\title{
Religionsfreiheit in Europa
}

\author{
Hans Michael Heinig"
}

Rezension zu Antje von Ungern-Sternberg, Religionsfreiheit in Europa. Die Freiheit individueller Religionsausübung in Großbritannien, Frankreich und Deutschland - ein Vergleich, Tübingen (Mohr Siebeck - Ius Ecclesiasticum, Band 86), 2008, XXIII, 404 Seiten, $79 €$. ISBN 978-3-16-149682-0

1. Die aus dem Amt scheidende deutsche Richterin am Europäischen Gerichtshof für Menschenrechte, Renate Jaeger, wurde unlängst von der Süddeutschen Zeitung (Ausgabe Nr. 236 vom 12. Oktober 2010, S. 6) gefragt, ob angesichts der unterschiedlichen religionsrechtlichen Systeme in Europa eine einheitliche europäische Perspektive auf die religiösen Konflikte in den europäischen Gesellschaften nicht an ihre Grenzen stoße. Ihr Antwort: Menschenrechte würden im EGMR „als etwas von der Religion Getrenntes empfunden“, mit der Konsequenz, „dass die Religionsferne unserer Urteile sehr viel evidenter ist als beim Bundesverfassungsgericht“. Deutschland bringe zwar den großen Kirchen, nicht aber anderen Religionsgemeinschaften wohlwollende Neutralität entgegen. Dieses Modell sei europaweit eine Ausnahme, erzeuge Diskriminierungen und sei den Kollegen am Gerichtshof, die nicht so kirchennah wie die Richter am Verfassungsgericht in Karlsruhe seien, nur schwer zu vermitteln.

Die Aussagen sind aus mehrerlei Gründen bemerkenswert. Sie sind unhistorisch, soweit sie die ambivalente Entstehungsgeschichte der Menschenrechte im Kontext christlich geprägter Gesellschaften eindimensional auflösen. Sie sind uninformiert, soweit sie übergehen, dass in Deutschland neben den beiden großen Kirchen auch alle anderen dazu bereiten Religions- und Weltanschauungsgemeinschaften an den Förderungs- und Kooperationsstrukturen des Grundgesetzes partizipieren. Solche Strukturen gibt es in unterschiedlicher Form zudem in weiten Teilen Europas. Unabhängig von staatskirchenrechtlichen Systemfragen wirft vor allem der Islam in seiner religiösen und organisatorischen Vielfalt (in ganz Europa) religionsrechtliche Integrationsprobleme auf. Seitens des deutschen Staates wird seit geraumer Zeit der klare Wille artikuliert, diese Probleme zu überwinden; dazu müssen die Muslime in Deutschland freilich auch die ihnen obliegende organisatorische Bringschuld erbringen. Von einer systematischen Diskriminierung der kleineren Religionsgemeinschaften in Deutschland kann deshalb schwerlich gesprochen werden. Die Einlassungen der Richterin, die vor ihrem Wechsel nach Straßburg zehn Jahre am Bundesverfassungsgericht tätig war, sind aber auch in rechtswissenschaftlicher Hinsicht erstaun-

* Prof. Dr. Hans Michael Heinig ist Inhaber des Lehrstuhls für Öffentliches Recht, insb. Kirchen- und Staatskirchenrecht an der Juristischen Fakultät der Georg-August-Universität Göttingen und im Nebenamt Leiter des Kirchenrechtlichen Instituts der EKD. 
lich. Denn die von der SZ angesprochenen methodischen und verfassungstheoretischen Probleme im Spannungsfeld von durch die EMRK europaweit garantierter Religionsfreiheit und unterschiedlichsten religionsrechtlichen Systemen in den fast 50 Signatarstaaten werden schlicht übergangen: Lässt sich ein gemeineuropäischer Besitzstand in Fragen der Religionsfreiheit ausmachen? Welche Standards in Fragen religiöser Freiheit und Gleichheit können von einem überstaatlichen Gerichtshof angesichts der von der Menschenrechtskonvention bewusst unangetastet gelassenen religionsrechtlichen Heterogenität in Europa gesetzt werden? Wie viel durch den EGMR erzeugter Anpassungsdruck ist nötig, wie viel noch zulässig? Welche Instrumente stehen zur Verfügung, das rechte Verhältnis zwischen der Durchsetzung gemeineuropäischer Mindeststandards und der Wahrung der religionspolitischen Souveränität der Unterzeichnerstaaten der EMRK zu wahren?

2. Wer an Fragen dieser Art interessiert ist, darf sich wertvolle Erkenntnisse versprechen, wenn er Antje von Ungern-Sternbergs Buch „Religionsfreiheit in Europa“ zur Hand nimmt. Die von Stefan Kadelbach betreute Dissertationsschrift bietet einen Vergleich der Freiheit individueller Religionsausübung in Großbritannien, Frankreich und Deutschland und setzt dabei einen starken Akzent auf die Rolle der EMRK für diesen Schutz. Sie reiht sich ein in eine Fülle neuerer Titel mit Interesse an europäischen Entwicklungen und/oder rechtsvergleichendem Ansatz auf dem Gebiet des Religionsrechts (insb. C. Walter, Religionsverfassungsrecht, 2006; S. Mückl, Europäisierung des Staatskirchenrechts, 2005). Innerhalb dieses Schriftenensembles gewinnt die Arbeit von Frau von Ungern-Sternberg ein eigenständiges Profil durch die Konzentration auf Fragen des individualrechtlichen Schutzes. Die thematische Selbstbeschränkung hat zur Konsequenz, dass Aspekte der korporativen Religionsfreiheit, freiheitsrechtlich fundierte Leistungs- und Förderungsansprüche sowie die vielfäligen sonstigen Bestimmungen des staatlichen und unionalen Religions- und Staatskirchenrechts zur Seite treten und nur insoweit gestreift werden, als sie für die individuelle Religionsausübungsfreiheit von besonderer Bedeutung sind.

Will man sinnvoll Rechtsvergleichung betreiben, muss man das Vergleichsmaterial beschränken, zugleich aber methodisch ausweisen, mit welchen Gründen die notwendige Auswahl vorgenommen wurde. Das ist im Grunde ein Dilemma - selten gelingt beides gleich überzeugend. Die von $v$. Ungern-Sternberg vorgenommene thematische Beschränkung auf Fragen der individuellen Religionsfreiheit erscheint vor dem Hintergrund des vorliegenden Schrifttums legitim - auch wenn damit die Gefahr verbunden ist, die komplexen religions(verfassungs)rechtlichen Regelungsgefüge samt ihrer spezifischen historischen, kulturellen, politischen und rechtssystematischen Kontexte in ihrer Bedeutung für die individuelle Religionsausübungsfreiheit nicht immer adäquat zu fassen zu kriegen. Begründungsbedürftig ist zudem die Auswahl der zu vergleichenden Rechtsordnungen. Hierzu finden sich bei von Ungern- 
Sternberg nur wenige Ausführungen. Die Autorin betont in der Einleitung die Unterschiede zwischen Deutschland, Frankreich und Großbritannien in „Tradition und Konzeption des Grundrechtsschutzes im allgemeinen und der Religionsfreiheit im besonderen“ (S. 3). Tatsächlich lebt das Buch in der Folge stark von der Spannung, die sich aus dem Kontrast einer staatskirchlichen, laizistischen und kooperativ-paritätischen religionsrechtlichen Tradition sowie aus den tiefgreifenden Unterschieden zwischen Frankreich, Großbritannien und Deutschland im Verständnis, in den Funktionen und in den Institutionen des Grundrechtsschutzes ergeben. So gesehen sind die Referenzordnungen glücklich gewählt. Freilich wäre eigens zu klären, ob diese Länderauswahl eingedenk der Unterschiede in der generellen Grundrechtskonzeption auch dem Bemühen um die Herausarbeitung gemeineuropäischer Standards dienlich ist. Über alle Signatarstaaten der Menschenrechtskonvention hinweg betrachtet, stehen Frankreich und Großbritannien in Fragen der der Grundrechtsbindung des Gesetzgebers und der Möglichkeiten ihrer gerichtlichen Durchsetzung vielleicht eher für Sonderwege und (ausgerechnet) das deutsche Grundgesetz (inzwischen) für einen europäischen Mainstream.

3. Die Arbeit von $v$. Ungern-Sternberg gliedert sich in sechs Kapitel. Der Aufbau folgt einem klassischen Schema: Geschichte, Völkerrecht und Unionsrecht werden einleitend abgehandelt (S. 7 ff.). Kapitel 2 widmet sich dem Schutz der Religionsausübungsfreiheit durch die EMRK. Dem folgen Länderberichte zu Frankreich (Kap. 3), Großbritannien (Kap. 4) und Deutschland (Kap. 5). Das Schlusskapitel dient dann dem Vergleich und der Synthesenbildung.

a) Über die Anlage des ersten Kapitels kann man stolpern. Geschichte und internationales Recht werden gemeinsam unter „Rahmen der Religionsausübung“ abgehandelt. Sind das nicht sehr verschiedene „Rahmen“ - die völker- und unionsrechtlichen Vorgaben einerseits und die Genesen der sozio-politischen Kontexte andererseits (in ihren jeweiligen Verquickungen von blutiger Konfessionsspaltung, Verfolgung religiöser Minderheiten, Tolerierungsstrategien und tatsächlicher Verwirklichung reziproker Anerkennung als Freie und Gleiche im Zuge der Ausbildung allgemeiner Grund- und Menschenrechtsstandards)? Mit solchen Fragen hält sich die Verfasserin nicht lange auf. Die Darstellung des „geschichtlichen Rahmens“ bietet gleichwohl einen knappen, aber kenntnisreichen Einblick in den historischen Resonanzraum des Themas. Das Völkerrecht jenseits der EMRK und das Unionsrecht werden hingegen etwas stiefmütterlich behandelt. Positiv gewendet könnte man auch sagen, dass sich die Verfasserin auf ihr eigentliches Thema konzentriert.

b) Den anschließenden Kapiteln zur EMRK und zu den drei Referenzstaaten liegt eine gemeinsame Matrix zugrunde, die im Vergleichsabschnitt im Detail explizit heraus präpariert wird, die die Darstellung vorher aber schon erkennbar prägt. Hierdurch kann der Eindruck entstehen, das Buch weise Redundanzen auf. Doch gewisse 
Wiederholungen sind notwendige Folge einer skrupelhaften Aufarbeitung des herangezogenen Materials, das eben zunächst länderbezogen entfaltet und dann noch einmal in einer systematischen Gesamtschau präsentiert wird.

Um unterschiedlichen Dimensionen der Religionsausübung nachgehen zu können, entwickelt von Ungern-Sternberg vier Typen religiöser Verhaltensweisen von Individuen (S. 5 und öfter): (I.) Gottesdienst und rituelle Handlungen, (II.) die Verkündigung religiöser Botschaften (Glaubensweitergabe), (III.) Verhaltensweisen, „die der Befolgung als zwingend empfundener religiöser Pflichten dienen“ (Gewissensfreiheit) sowie (IV.) die Beachtung sonstiger religiöser Bräuche im Alltag (religiöse Lebensführung). Jeder Typus kennt Subtypen, z.B. für die Gewissensfreiheit das Recht zur Kriegsdienstverweigerung, sonstige Verpflichtungen gegenüber dem Staat sowie die Freiheit zum Mitwirken bei bioethisch umstrittenen Handlungen oder für die Freiheit zur Glaubensweitergabe die Unterscheidung zwischen Meinungsäußerung gegenüber Dritten und Kindererziehung. Mit diesen feingliedrigen Differenzierungen im Hintergrund befragt die Autorin unterschiedliche Normkomplexe divergierender Normhierarchie und -typen, also Verfassungsrecht, Gesetzesrecht, Richterrecht, gebundenen Verwaltungspraxis, daraufhin, welche Verhaltensweise wie intensiv wodurch geschützt und welche Grenzen in den Rechtsordnungen gezogen werden. Dabei unterscheiden die Ausführungen zwischen dem subordinationsrechtlichen Freiheitsschutz gegenüber dem Staat und Schutzwirkungen im Privatrecht, wobei hier nochmals zwischen Arbeitsrecht, freiwillig eingegangenen Verpflichtungen und sonstigem Privatrecht (wie dem Erb- und Deliktsrecht) differenziert wird. Diese aufwendige Typen- und Subtypenbildung ist überzeugend konzipiert und führt zu tragfähigen Vergleichsergebnissen.

Einleitend wird im jedem Kapitel auf die Besonderheiten der Referenzrechtsordnung eingegangen. Im Anschluss werden die relevanten Normen vorgestellt und die Grundzüge der (höchst- wie der sonstigen fachgerichtlichen) Rechtsprechung zu den einzelnen Themenfeldern skizziert. Auf diese Weise bewältigt von Ungern-Sternberg große Informationsmengen. Die Zahl der berücksichtigten Entscheidungen aus der jeweiligen Rechtsprechung ist beeindruckend. Sie behandeln Einzelfragen wie die nach der Schulbefreiung in öffentlichen Schulen für einzelne Fächer und Tage oder nach der Zulässigkeit religiös konnotierter Kleidung im öffentlichen Dienst und in der Privatwirtschaft ebenso wie die großen Grundsatzprobleme. Die da wären: Was ist Religion aus freiheitsrechtlicher Perspektive überhaupt? Wie weit reicht der Schutz der Religionsausübung? Wie geht man mit religiös motivierten Handlungen um, deren religiöse Bedeutung nach außen für Dritte nicht ohne weiteres erkennbar ist? Meint Freiheit der Religionsausübung eine Art religiöse Handlungsfreiheit oder ist ein spezifischer Bezug zu den religiösen Überzeugungen geltend zu machen bzw. eine besondere Form der Handlung an den Tag zu legen (Kultus etc.)? Ist auf den 
Glauben des einzelnen abzustellen, selbst wenn dieser in der relevanten Einzelfrage von der Gemeinschaft, der er sich zugehörig erklärt, abweicht? Oder markiert das religiöse Kollektiv den grundrechtlichen Schutz individueller Religionsausübung? Sind nur Handlungen geschützt, die vorzunehmen der Gläubige meint gezwungen zu sein? Oder zählen auch Motive religiöser Traditionspflege? Und schließlich: Wie verhalten sich allgemeines Gesetz, d.h. die vom demokratischen Gesetzgeber ohne diskriminierende Absicht und besonderem Religionsbezug erlassenen Regelungen, zur individuellen Betroffenheit in der Religionsausübung?

c) Ein wichtiger Nebenstrang der Arbeit widmet sich der Bedeutung von Diskriminierungsverboten für den effektiven Schutz der Religionsausübung. Großbritannien bietet von Ungern-Sternberg dazu reichlich Anschauungsmaterial. Weniger intensiv leuchtet sie hingegen aus, wie Antidiskriminierungsrecht den sozialproduktiven Nutzen religiöser Freiheit auch beschädigen kann. Nicht ganz zufällig dazu ein Beispiel aus Frankreich: Dort wird gegenwärtig das Votum der französischen Antidiskriminierungsbehörde diskutiert, nach dem eine privat betriebene Kinderkrippe - in einer Banlieue-Siedlung gelegen und integrations- wie arbeitsmarktpolitisch ein Vorzeigeobjekt - die Ganzkörperverschleierung einer Erzieherin dulden muss. Begründung der Behörde: da die private Einrichtung nicht dem staatlichen Laizitätsgebot unterworfen sei, dürfe sie die Religionsfreiheit der Mitarbeiter nicht diskriminierend einschränken. Da wundert nicht, dass in Frankreich über ein generelles Burka-Verbot nachgedacht wird: Man kann das Religionsrecht auch so unglücklich konstruieren, dass staatlich verordnete Religionsverdrängung oder schleichende Islamisierung als die einzig möglichen Alternativen erscheinen, statt auf die konstruktive Gestaltungskraft gesellschaftlicher Freiheit zu setzen.

4. In der Gesamtschau zeigt die Arbeit von $v$. Ungern-Sternberg anschaulich, dass die Referenzrechtsordnungen in Fragen des Schutzes der individuellen Religionsausübung bis in den Details der Einzelfragen vor vergleichbaren lebensweltlichen Problemen und rechtsdogmatischen Herausforderungen stehen. Diese Homogenität über alle Systemunterschiede hinweg steht für einen Grundbestand geteilter Religions- wie Rechtskultur in den europäischen Gesellschaften. Die Religionsrechtskultur wird jedoch ebenso durch unterschiedliche Problemlösungen geprägt. Mal sind die Differenzen grundsätzlicher Natur, mal zeigen sie sich auch nur in Nuancen. Der große Verdienst der Arbeit von $v$. Ungern-Sternberg ist es, mit Sinn für die einzelnen Problemfelder beides, sowohl die Gemeinsamkeiten wie auch die Unterschiede, entlang tragfähiger thematischer Systematisierungen aufzubereiten und offen zu legen.

Gerne wüsste man mehr über die tieferliegenden Gründe: Warum wird wann welche Lösung gewählt? Welche Alternativen werden diskutiert? Welche Pfadabhängigkeiten bestehen? Welche gesellschaftlichen Entwicklungen und Konflikte bewirken Veränderungen? Um Fragen dieser Art beantworten zu können, sind sozio-kulturelle 
Kontextvergleiche ebenso notwendig wie eine intensive Auseinandersetzung mit dem wissenschaftlichen Schrifttum, sind theoretische Einbettungen ebenso vorzunehmen wie rechtsdogmatische Systematisierungen. Die Arbeit von $v$. Ungern-Sternberg bietet in dieser Hinsicht keine umfassenden Antworten, aber doch immerhin gewisse Orientierungen. Der Einfluss religiöser Konflikt- als religionsrechtlicher Identitätsfindungsgeschichte wird ebenso erkennbar wie der der Korrelation von Demokratieund Grundrechtskonzeptionen. Doch weil sich von Ungern-Sternberg auf Normtext und Rechtsprechung konzentriert, dringt sie zu wichtigen Problemen nicht immer vor: So wäre etwa der Frage nachzugehen, ob Konzeptionen der Schutzbereichsbeschränkung sich an kohärenten Kriterien orientieren, die eine willkürfreie, faire und freiheitsadäquate Handhabung der einzelnen Fälle ermöglichen. Schaut man sich die Entscheidungen, die eine restriktive Linie in der Schutzbereichsbestimmung fahren an, wird man daran zweifeln dürfen.

Pointierte Kritik solcher Art findet sich bei von Ungern-Sternberg selten. Dies fördert den Eindruck hoher wissenschaftlicher Sachlichkeit, geht aber zuweilen zulasten der analytischen Tiefenschärfe. So hätte die Rechtsprechungspraxis des Europäischen Gerichtshofs für Menschenrechte durchaus eine distanziertere Betrachtung verdient. Der Gerichtshof unterliegt als überstaatliches Gericht mit der begrenzten Aufgabe, menschenrechtliche Mindeststandards in den Signatarstaaten durchzusetzen, besonderen Arbeitsbedingungen und Funktionszwängen. Diese bedürfen einer systematischen, für die Rechtsfindung relevanten Aufarbeitung. Daran fehlt es in Straßburg zuweilen - man überlege nur, wann und wie die Figur des margin of appreciation verwendet findet (oder auch nicht). Umso schwerer wiegt, dass der Gerichtshof sich lange Zeit bei der Prüfung einer Verletzung von Art. 9 EMRK schwer damit tat, eine sinnvolle, d.h. die Rationalität und Vorhersehbarkeit der Entscheidung sichernde Linie in der Frage auszubilden, auf welcher Prüfungsstufe mit welchen Argumentationsfiguren für Fragen der Religionsausübungsfreiheit typische Probleme abgearbeitet werden. Mittlerweile ist dem Mangel dogmatischer Grundausstattung abgeholfen - die Rechtsprechung hat nachgesteuert.

Zugleich wirft die jüngere Spruchpraxis des EGMR neue Fragen auf. $v$. UngernSternbergs Dissertation zeigt in ihren facettenreichen Länderstudien, dass Europa in Fragen der Religionsfreiheit von einer prekären Balance zwischen Rechtseinheit und Lösungsvielfalt geprägt ist. In der Vergangenheit hat der EGMR das Gleichgewicht zwischen einheitlichem Mindestschutz und signatarstaatlicher Varianz bewusst unangetastet gelassen. Diese Selbstbeschränkung war gleichsam in das Erbgut der EMRK eingelassen. Ob Mutation oder Evolution: Zunehmend scheint sich Straßburg zu einem gesamteuropäischen Paraverfassungsgericht zu entwickeln, das nicht nur Mindeststandards durchsetzt, sondern für sich in Anspruch nimmt, vielfältige Abwägungsentscheidungen bis in detaillierte Verästelungen hinein für ganz 
Europa treffen zu können. In Fragen der Religionsfreiheit sprechen etwa die jüngsten Entscheidungen des EGMR zum Schulkreuz in Italien oder zum kirchlichen Arbeitsrecht in Deutschland (aus der Zeit nach dem Erscheinen des hier besprochenen Bandes) für einen solchen Trend. Wohlgemerkt: in der Sache vermögen die gefundenen Ergebnisse durchaus zu überzeugen. Doch die Rechtsprechung des EGMR hat sich nicht nur am Maßstab der Sachgerechtigkeit, sondern auch an dem der funktionalen Richtigkeit und demokratischen Akzeptanz messen zu lassen. Insoweit stellt sich schon die im eingangs erwähnten SZ-Interview aufgeworfene und von der deutschen Richterin am EGMR, Renate Jaeger, unbeantwortet gelassene Frage, ob es dem EGMR gut zu Gesicht steht, starken Homogenisierungsdruck in Fragen des Religionsrechts zu erzeugen. Jaegers Einlassungen wirken da fast wie eine Drohung: Religionsferne statt kulturelle Vielfalt sollen die Religionsfreiheit in Europa bestimmen. Antje von Ungern-Sternbergs fundierte und kompetente Darstellung zeigt, dass der Gerichtshof mit einer solchen Agenda der Religionsfreiheit in Europa nicht gerecht würde. 